Peteet, J. R. (1981) Issues in the treatment of religious patients. American Journal of Psychotherapy, 35, 559-564.

TrUAX, C. B. (1964) Current research in psychotherapy. Canada's Mental Health, 12, 5-9.

A. Aziz A. Salama

Mercer University School of Medicine

Macon

Georgia

USA

SIR: El-Sherbini \& Chaleby (Journal, March 1992, $160,425)$ feel that exploratory psychotherapy has little to offer in Arab culture. However, if one deletes the culture-specific words from their letter, their comments about the cognitive processes of their patients could be applied to the majority of the UK population as judged from the reflective, articulate middle-class backgrounds of most psychiatrists. Like the Arab-speaking world this is also reflected linguistically in the use of the simple concrete AngloSaxon derived part of the English language spoken by the majority of people in the UK. The idea that exploratory psychotherapy is, therefore, appropriate for only a small proportion of patients is still an issue as alive in Western Europe as it is in the Middle East.

A whole society in itself is unlikely to operate in a completely pre-formal way, witness the subleties of Islamic mysticism, but it may be that cultures vary somewhat in the proportion of their population who think concretely.

Finally, a different perspective on the subject might be that it is the task of exploratory psychotherapy to re-start the developmental process that opens up the possibility of a more reflective, less dichotomous and concrete way of thinking. Therapeutically this is commonly expressed as trying to reduce the mechanisms of rejection, splitting, etc., to negotiate the depressive position, but Piaget's or Erickson's terms could fit the model in exactly the same way.

Department of Psychological Medicine

Paul Mallett University of Wales College of Medicine Heath Park

Cardiff CF 4 XN

\section{Lithium augmentation in antidepressant-resistant patients}

SIR: Flint (Journal, May 1992, 160, 710) raises two methodological issues for further discussion and thereby casts doubt on the value of meta- or quantitative analysis of clinical trials. The issue of how long treatment should be continued before a patient can be considered treatment refractory is, of course, important. However, the evidence that treatment must be continued for six weeks before a judgement can be made is not yet convincing. Quitkin et al (1984) are widely cited in this regard. Their study consisted of a literature survey, which concluded that there was not adequate evidence up to that point to give a judgement, followed by a summary of three of their own trials. These studies were all in out-patients with mean Hamilton Rating Scale for Depression (HRSD; Hamilton, 1960) scores at trial entry under 16. Although it is possible to interpret their results as showing a 'response' of $25 \%$ of the patients between week four and week six of treatment, the change in mean (s.d.) HRSD scores underpinning this conclusion was from $9.6(4.5)$ to $8.2(5.6)$. The study by Georgotas et al (1986) was performed in elderly patients with a slow build-up of the dose of medication; it has no clear bearing either way on whether or not six weeks is a better time than four weeks to assess refractoriness. The studies we reviewed all showed levels of depression that were moderately severe (HRSD ratings above 20) and most of the patients had been depressed for more, often much more than three weeks; the implication that "many" were treated for only three weeks is misleading. We join with Quitkin et al (1984) in a plea for more evidence as to the length of time necessary to decide treatment efficacy but we believe it premature to conclude with Dr Flint that it is incumbent on clinicians "to persist with the original treatment" for more than four weeks.

The second issue relates to the definition of treatment response. It is one of the advantages of quantitative analysis that different outcome criteria can be used to define treatment effects within individual studies in a way that allows comparison between different studies. It is true that, for example, a $50 \%$ reduction in HRSD ratings is not the same as clinical remission. However, we would be surprised if Dr Flint can prove that they are not usually related.

In conclusion, we recognise the conventional argument that meta-analysis is limited by the quality of the data being analysed; unfortunately, qualitative analysis of clinical trials is even more limited by its methodology, or rather the lack of it. The greater danger with qualitative critiques of papers is that perfectly good evidence can be discounted in favour of the particular trial or method of treatment that a reviewer chooses to favour. Completely incompetent clinical trials are perhaps published in refereed journals less commonly than it is academically convenient to pretend. Quantitative critiques put all trials on the same footing; inexplicable quantitative differences will then expose trials which really are misleading. One trial was, in fact, so revealed in 
our own review. Just as importantly, quantitative analysis also allows summary conclusions to be reached on the basis of as many cases as possible. It is the number of cases entered in randomised studies that determine the confidence we can have in the findings. An overview of treatment studies in breast cancer illustrates the point $(n=75000)$, and previous controversy over the same treatments exemplifies what may be described as the qualitative fallacy (Early Breast Cancer Trialists' Collaborative Group, 1992). The latter publication shows what can be done when clinicians take treatment issues seriously. We will be delighted if our conclusions serve as a stimulus to further studies on patients defined more strictly for refractory illness. However, the existing data from randomised trials, together with a good deal of more anecdotal evidence which should not be discounted, support the view that lithium augmentation is an effective manoeuvre in patients who have not responded to a tricyclic antidepressant.

Early Breast Cancer Trialists' Collaborative Group (1992) Systemic treatment of early breast cancer by hormonal, cytotoxic, or immune therapy: 133 randomised trials involving 31,000 recurrences and 24,000 deaths among 75,000 women. Lancet, $339,1-15$.

GeORGOTAS, A MCCUE, R. HAPWORTH, W et al (1986) Comparative efficacy and safety of MAOls versus TCAs in treating depression in the elderly. Biological Psychiatry, 21, $1155-1166$.

Hamilton, M. (1960) A rating scale for depression. Journal of Neurology. Neurosurgery and Psychiatry, 23, 56-62.

QuirkIN, F. M., RABKIN, J. G., Ross, D., et al (1984) Duration of antidepressant drug treatment. What is an adequate trial? Archives of General Psychiatry, 41, 238-245.

G. M. GoodwIN

F. G. M. SouzA

MRC Brain Metabolism Unit

Royal Edinburgh Hospital

Morningside Park

Edinburgh EHIO 5HF

Mood Disorders Unit

M.-P. Austin

Prince Henry Hospital

2036 Sydney

Australia

\section{Who benefits from ECT?}

SIR: The casual or unsophisticated reader of the recent article by Buchan et al (Journal, March, 1992, $160,355-359)$, upon encountering the statements in the abstract that "patients who were neither retarded nor deluded did not benefit significantly from real as opposed to simulated ECT", and later in the summary that "real ECT does not appear to be effective in non-retarded, non-deluded patients", might not realise that the authors did not actually determine whether any non-retarded, non-delusional patients were ECT-responders. In fact, Buchan et al have simply demonstrated the truism that removing ECT-responders from a sample of depressives leaves a subsample of ECT non-responders.

Only randomised prospective comparisons of genuine v. sham ECT, with stratification of subjects by the clinical predictor variables of interest (e.g. presence of delusions or retardation), can definitely answer the question: "Who benefits from ECT?".

RICHARD ABRAMS

Department of Psychiatry and Behavioral Sciences 3333 Green Bay Road

North Chicago

Illinois 60064-3095

USA

\section{Do benzodiazepines interfere with the action of ECT?}

SIR: Cohen \& Lawton (Journal, April 1992, 160, 545-546) suggest that the presence of benzodiazepine drugs may interfere with the ability of the brain to respond to bilateral ECT. I would point out that, in experimental animals at least, there is indeed evidence for this.

When electroconvulsive shocks (ECS) are given to mice in a manner somewhat similar to the clinical administration of ECT ( 5 ECS given spread out over 10 days to anaesthetised animals) various changes occur in neurotransmitter function. These include enhanced behavioural responses to drugs stimulating dopamine and 5- $\mathrm{HT}_{2}$ receptors and an attenuated response to the sedative effects of the $\alpha_{2}$ adrenoceptor agonist clonidine (for review see Green \& Nutt, 1987) and it has been proposed that some of these changes could be associated with the antidepressant action of ECT (Green \& Nutt, 1987).

When diazepam was given before each ECS, the dopamine and 5- $\mathrm{HT}_{2}$-receptor-mediated behavioural changes no longer occurred (Green \& Mountford, 1985). This was clearly not due to any modification by the benzodiazepine of the convulsant effect of the ECS both because no obvious modification was seen to occur and, most critically, because the same effect was seen when the diazepam was given 5 minutes after the ECS administration. This effect of diazepam also appeared to be due to a specific action at the benzodiazepine receptor-binding site in the brain because the selective benzodiazepine antagonist flumazenil blocked the effect of diazepam on the ECS-induced changes (Green \& Mountford, 1985).

It is always difficult to speculate on the relevance of animal experimentation to clinical practice. 